Ecole d'Eté Systèmes Optiques

\title{
Optiques actives et adaptatives
}

\author{
G. Bourdet
}

Laboratoire d'Optique Appliqué, Ecole Nationale de Techniques Avancées, Ecole Polytechnique, F-91120 Palaiseau, France \begin{abstract}
moderne, Parmi les nouveaux domaines de l'optique classique intéressant et le plus prometteur. Dans ce domaine viennent prendre place tous les grands projets de l'astronomie moderne, le déploiement de grands télescopes allégés dans l'espace, la réalisation au sol de télescopes de grands diamètres corrigés de leurs propres déformations ou de la turbulence atmosphérique, la mise en place de matrices de télescopes permettant la synthèse $\mathrm{d}^{\prime}$ ouverture ou l'optimisation des grands interféromètres stellaires.

L'histoire nous apprend que, pour défendre Syracuse assiégé par la flotte romaine de Marcellius en 215 avant J.C., Archimède eut l'idée de focaliser sur les bateaux en bois des assiegeants la lumière du soleil en la faisant se réfléchir sur les boucliers des soldats qui défendaient la ville. Plus de deux millénaires aprés, et pour la même application, des dispositifs à miroirs ont été étudiés et réalisés dans le but de pouvoir focaliser la lumière émise par des lasers de trés forte énergie sur des cibles agressives malgré la turbulence atmosphérique ou la turbulence induite par l'échauffement provoqué par le faisceau lui-même.
\end{abstract}

Dans un domaine plus modeste, nous pouvons aussi considérer les nombreux dispositifs grand public qui permettent à des photographes non expérimentés de pouvoir réaliser de trés beaux souvenirs de voyage.

Compte tenu de l'ampleur du sujet, nous restreindrons notre exposé à une analyse générale des dispositifs à miroirs utilisés pour corriger les effets de la turbulence atmosphérique tels qu'on commence à les rencontrer en astronomie. Nous exposons, tout d'abord, un modèle capable de représenter simplement la turbulence atmosphérique. Nous analyserons ensuite les diverses techniques capables de déformer à volonté une surface d'onde optique. Nous nous intéresserons ensuite aux dispositifs utilisés pour l'analyse en temps réel des surfaces d'onde.

\section{La turbulence atmosphérique.}

L'atmosphère peut être considérée comme un milieu inhomogène où $l^{\prime}$ indice de réfraction est une fonction aléatoire du temps et de l'espace. On peut le représenter comme un ensemble de volumes de tailles variées dans lesquels l'indice prend des valeurs qui varient autour d'une valeur moyenne. Lorsque une onde 
optique cohérente traverse un tel milieu, nous assistons à une perturbation aléatoire de la phase de l'onde transmise et donc les interférences de l'onde diffractée au niveau d'un détecteur vont donner lieu à des fluctuations aléatoires de l'intensité. Ce phénomène peut être analysé en terme d'espace en considérant le rapport entre le diametre du faisceau $D$ et la taille de ces inhomogénéités d'indice 1 .

De nombreux travaux ont été consacrés a l'étude de la transmission des ondes à travers 1'atmosphère. On suppose que la turbulence atmosphérique est caractérisée par une fonction de structure $d^{\prime}$ indice $c_{n}(r)$ ou $r$ est l'épaisseur de la couche d'atmosphère considérée. 'L'écart quadratique moyen des fluctuations de phase entre deux points distants de $\rho$ s'écrira :

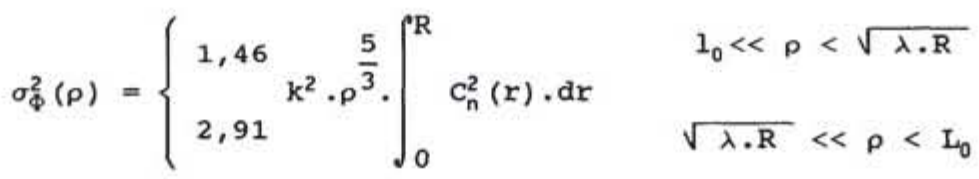

ou $k$ est le nombre $d^{\prime}$ onde $\left(\frac{2 . \pi}{\lambda}\right), \quad R$ est la distance entre l'émetteur et le récepteur, $r$ est le rayon vecteur de composantes $x, y$ et $z$ et $I_{0}$ et $L_{0}$ sont, respectivement, les dimensions des plus petites et des plus grandes inhomogénéités. Si la structure de la turbulence, ne varie pas le long de la propagation, l'intégrale se réduit à $C_{n}^{2} \cdot R$.

Deux cas limites peuvent se présenter :

- Les inhomogénéités ont des dimensions trés supérieures à l'ouverture de l'instrument. Dans ces conditions, le faisceau est dévié globalement et l'écart quadratique moyen de cette déviation est donné par :

$$
\sigma_{\theta}=\frac{\sigma_{\Phi}}{\mathrm{k} \cdot \rho}
$$

- Le front d'onde est distordu de telle sorte que $\rho$ satisfasse la condition :

$$
\sigma_{\Phi}(\rho)>\pi
$$

La corrélation de phase est alors nulle entre les deux points considèrés. Ceci nous amène à définir une largeur transverse de cohérence $\rho_{0}$ telle que :

$$
\sigma_{\Phi}\left(p_{0}\right)=\pi
$$

cohérente.

$$
\rho_{0}<D \quad \text { La source } n^{\prime} \text { apparait plus comme une source }
$$

$$
\rho_{0}>D \quad \text { Le faisceau est dévié globalement. }
$$

Les fluctuations d'amplitude au niveau d'un récepteur peuvent aussi être définies à partir de la même fonction de structure d'indice : 


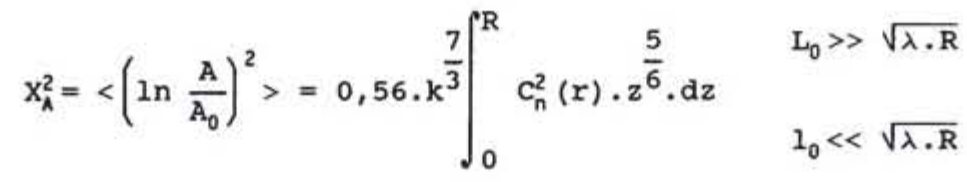

Les figures $1-a$ et $1-b$ montrent des valeurs de la largeur transverse de cohérence pour trois longueurs $d$ 'onde en fonction de la distance entre la source et le récepteur ainsi que les valeurs de $x_{A}$ pour ces mêmes longueurs $d^{\prime}$ onde.

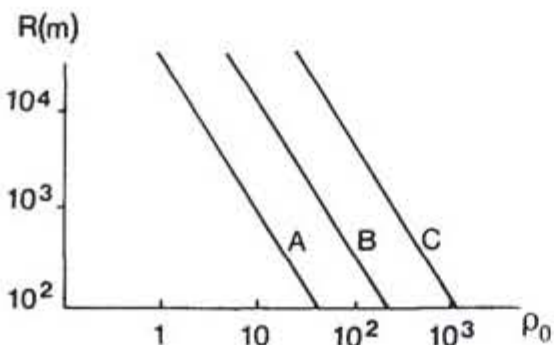

Fig.1-a

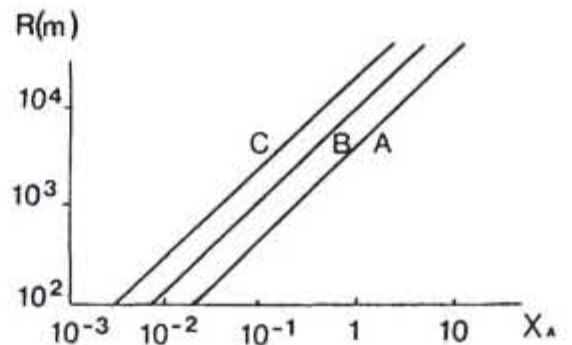

Fig.1-b

Largeur transverse de cohérence et valeur RMS des fluctuations $d^{\prime}$ amplitude pour une turbulence moyenne.
A: $\lambda=0,7 \mu \mathrm{m}$
B: $\lambda=3 \mu \mathrm{m}$
C: $\lambda=10 \mu \mathrm{m}$

\begin{tabular}{|c|c|c|}
\hline & $\rho_{0}$ & $\mathrm{X}_{\mathrm{A}}$ \\
\hline Turbulence faible & $\times 0,03$ & $\times 0,2$ \\
Turbulence forte & $\times 6,9$ & $\times 12,5$ \\
\hline
\end{tabular}

Considérons, maintenant, une onde distordue de la quantité $\phi(\rho, \theta)$ par rapport à une onde sphérique de référence. Le rapport de l'énergie encerclée dans un cercle de rayon a autour du point de focalisation de l'onde de référence à l'intensité créée en ce point par cette onde s'écrira (rapport de STREHL) :

$$
i(P)=\frac{1}{\pi^{2}} \cdot\left|\int_{0}^{1} \int_{0}^{2 \pi} \exp i k \Phi(\rho, \theta) \cdot d \rho \cdot d \theta\right|^{2}
$$

Pour de faibles aberrations, ce rapport peut s'écrire : 


$$
i(\mathrm{P}) \sim 1-\left[\frac{2 \pi}{\lambda}\right]^{2} \cdot \sigma_{\Phi}^{2}
$$

où $\sigma_{\Phi}^{2}$ est l'écart quadratique moyen de la fluctuation de phase. Nous voyons donc, que pour un écart de phase égal à $\lambda / 10$, l'énergie encerclée est réduite de $40 \%$. Cette grande sensibilité aux aberrations est une motivation importante pour l'usage des optiques actives correctrices. Cette correction pourra, éventuellement, corriger toutes les aberrations qui dégradent la surface $d$ 'onde. Ces aberrations peuvent être d'origine atmosphérique ou il peut s'agir des abérrations propres de l'instrument ou de celles provoquées par des déformations mécaniques du système.

Un dispositif capable de corriger en temps réel la surface d'une onde optique comprendra :

localement la surface d'onde.

- Un dispositif d'action qui permettra de deformer

- Un dispositif d'analyse capable de mesurer en temps réel les défauts.

- Un dispositif de réaction qui élaborera, à partir des informations fournies par le dispositif d'analyse, des signaux capables d'agir sur le premier système en vue d'obtenir le résultat souhaité.

\section{$\underline{2^{\circ}-\text { Miroirs correcteurs de surface } d^{\prime} \text { onde. }}$}

Nous allons introduire une représentation simplifiée de la déformation de la surface d'onde à corriger. Considérons le problème à une dimension et appelons $f(x)$ la déformation. Au voisinage d'un point d'abscisse a et dans un domaine de largeur $b$, cette fonction peut se mettre sous la forme :

$$
f(x)=f(a)+x \cdot f^{\prime}(a)+\frac{x^{2}}{2} \cdot f^{\prime \prime}(a)+\ldots \ldots
$$

ou $f^{\prime}(a)$ est la pente de la déformation au point $x=a, 1 / f^{\prime \prime}(a)$ est la courbure en ce point, .... Une description plus complete en terme de polynômes de zernique permet $d^{\prime}$ identifier des termes d'aberration tels que le basculement, la défocalisation, l'aberration sphérique, la courbure, la coma, l'astigmatisme, ...

Nous pouvons obtenir une reconstition approximative de la forme de l'onde par un échantillonnage convenablement choisi de période a :

$$
f(x)=\sum_{m}\left\{f(m a)+(x-m a) \cdot f^{\prime}(m a)+\frac{(x-m a)^{2}}{2} \cdot f^{\prime \prime}(m a) \cdots\right\}
$$

où chaque échantillon a une extension transverse ma $\mp \frac{b}{2}$. 
1-a Miroirs segmentes en translation (piston - Fig.5a).

Nous supposons, dans un premier temps, que tous les termes dépendant de $\mathrm{x}$ sont négligeables dans $1^{\prime}$ intervalle $\left[\mathrm{ma}-\frac{\mathrm{b}}{2}, \mathrm{ma}+\frac{\mathrm{b}}{2}\right]$.

$$
\frac{\left(m a \mp \frac{b}{2}\right)^{n}}{n !} \cdot f^{(n)}(m a)<\lambda
$$

Dans ces conditions, les déformations de la surface d'onde au voisinage du point $x=m a$ peuvent être assimilées à $f(m a)$, La correction pourra être effectuée par une mosalque de mirairs de petites dimensions transverses se déplaçant parallèlement à eux mêmes. Dans ce dispositif, chaque miroir diffracte la portion d'onde qu'il réfléchit dans un lobe d'ouverture $\frac{\lambda}{b}$ et l'image est reconstituée en remettant en phase la lumière dè ces différents lobes.

En général, et du fait des discontinuités à la limite de deux miroirs adjacents, la tache reconstituée présente des lobes secondaires. De plus, ce type de miroir perd beaucoup de son efficacité lorsque l'objet $n^{\prime}$ est pas situé sur son axe. Ces deux comportements sont montrés sur 1'enregistrement représenté sur la figure 2. Il s'agit de la tache de diffraction d'une ligne de 5 miroirs carrés asservis pour focaliser la lumière issue d'un laser a $\mathrm{CO}_{2}$ sur une cible. Lorsque la cible est sur 1'axe de l'instrument, la tache de diffraction présente des lobes secondaires résultants de la discontinuité entre les miroirs. Lorsque la cible se déplace hors de l'axe, la figure de diffraction reste enveloppée par la figure de diffraction du miroir élémentaire. On observe alors une diminution du pic central et une augmentation d'un des lobes secondaires. Pour éviter ce défaut, il est possible de compléter ce type de dispositif par un miroir unique capable de compenser les basculements d'ensemble de la surface d'onde. On rencontre le même type de problèmes en ce qui concerne la focalisation et on peut aussi utiliser un troisième miroir en série avec les deux premiers et dédié à compenser la sphérisation de la surface d'onde. 


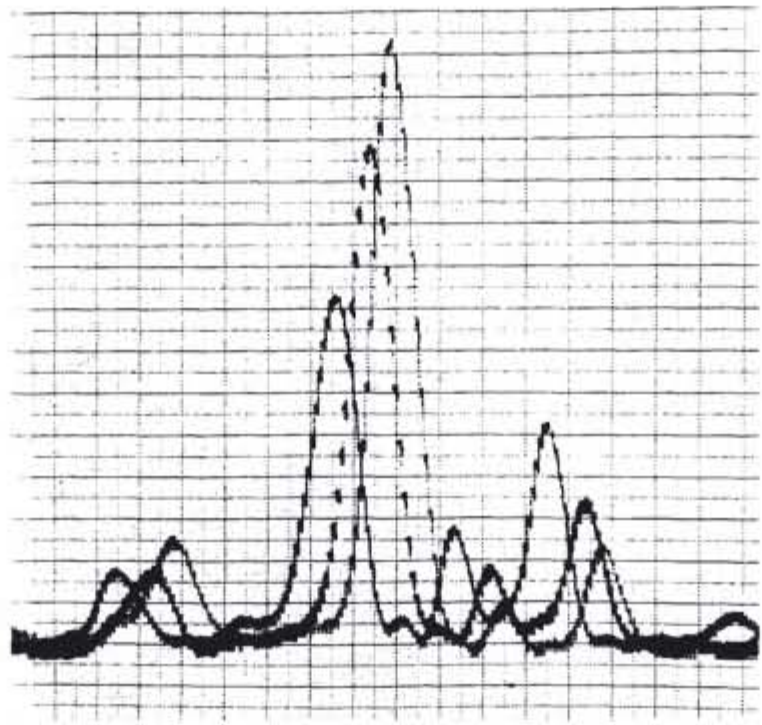

Maxima secondaires dans la tache de diffraction pour un dispositif à miroirs segmentés en translation.

Fig. 2

2-b Miroirs segmentés à translation et basculement (piston and tilt - Fig.5b).

(b) Pour le même échantillonnage de pas a, la quantité $\left(\operatorname{ma} \frac{b}{2}\right) \cdot f^{\prime}(m a)$ peut ne plus être négligeable. Nous pouvons alors compenser le basculement local de la surface d'onde par un basculement de chaque miroir élémentaire ce qui conduit à un dispositif où chaque miroir est animé par 3 degrés de liberté. Ce type de dispositif présente les mêmes inconvénients que le précédent en ce qui concerne les lobes secondaires introduits par les discontinuités entre deux miroirs adjacents. Il nécessite cependant 2,5 fois moins de miroirs que le précédent pour une correction équivalente.

2-c Miroirs continument déformables.

La mise en oeuvre du dispositif précédent exige 3 degrés de liberté pour 2,5 fois moins de miroirs que le premier dispositif décrit. Un dispositif beaucoup plus efficace consiste en un miroir continoment déformable qui serait déformé par une mosaïque de moteurs agissant suivant un maillage convenablement choisi. Dans ce cas, la courbure locale de la surface d'onde serait aussi corrigée et le problème de l'effet des discontinuités entre deux miroirs adjacents est supprimé. Les moteurs peuvent agir soit en fixant la position du point du miroir sur lequel ils agissent (Fig. $5 \mathrm{c}$ ), soit en $y$ exerçant une force qui sera équilibrée par les couples exercés par la rigidité de la membrane 
(Fig.5d). Certains dispositifs utilisent des moteurs disposés parallelement à la membrane et qui induisent des courbures locales (Fig.5e).

Des calculs ont montré les mérites de ces trois solutions. Sur la figure 3 sont traçés l'écart quadratique moyen de l'erreur résiduelle en fonction de la quantité $\left[\frac{\rho_{\mathrm{e}}}{\rho_{\mathrm{o}}}\right]^{\frac{5}{3}}$ ou $\rho_{\mathrm{e}}$ est $1^{\prime}$ écartement entre deux moteurs adjacents et $\rho$ la longueur transverse de cohérence. Dans le cas des miroirs déformables, la forme de la déformation du miroir autour de chaque point d'appui a été supposée gaussienne.

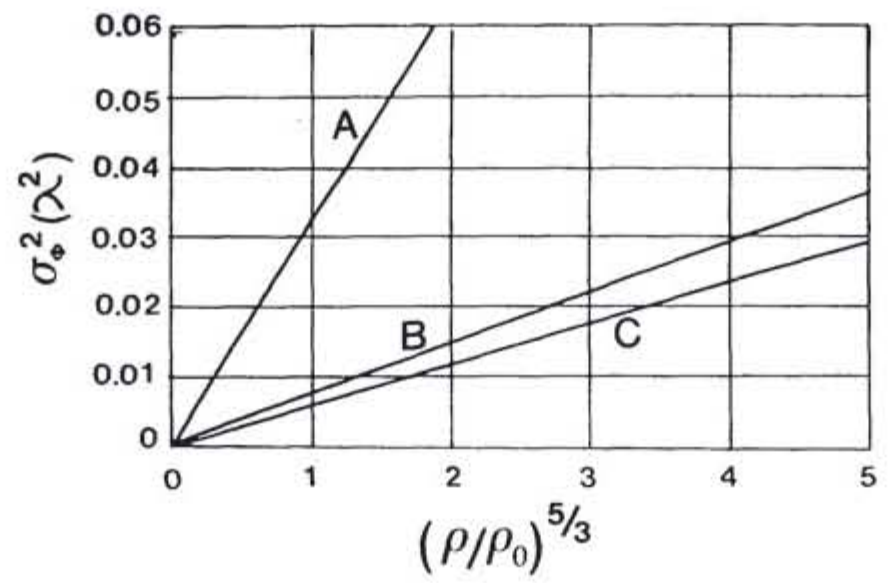

Erreur résiduelle en fonction du rapport $\left(\frac{\rho_{e}}{\rho_{0}}\right)^{\frac{5}{3}}$ pour trois types

de miroirs -A Miroirs segmentés en translation -B miroirs segmentés en translation et basculement -C Miroir continoment déformable à déformée gaussienne.

Fig. 3

Des miroirs déformables ont été réalisés sous différentes formes. Le plus souvent, il s'agit d'une plaque mince d'un matériau stable et pouvant' être optiquement poli. A l'opposé de la face polie agissent un certain nombre de moteurs suivant un maillage adéquat. La déformation de la plaque peut être caractérisée par sa forme et par un facteur de couplage qui est le rapport entre l'amplitude de la déformation au point d'appui le plus proche et la déformation là où est appliquée la force. A partir de ces données, il est possible de calculer les modes de deformation de la plaque pour toutes les sollicitations des moteurs. Sur la figure 4 représente l'enregistrement de la déformée d'un miroir. Ce miroir est constitué d'une plaque de Molybdène encastrée suivant un diamétre de $16 \mathrm{~cm}$ et activé par 7 
moteurs dont un est situé au centre du miroir et les six autres sont disposés suivant un cercle. Le moteur central est activé et le plan de l'enregistrement passe suivant son axe.

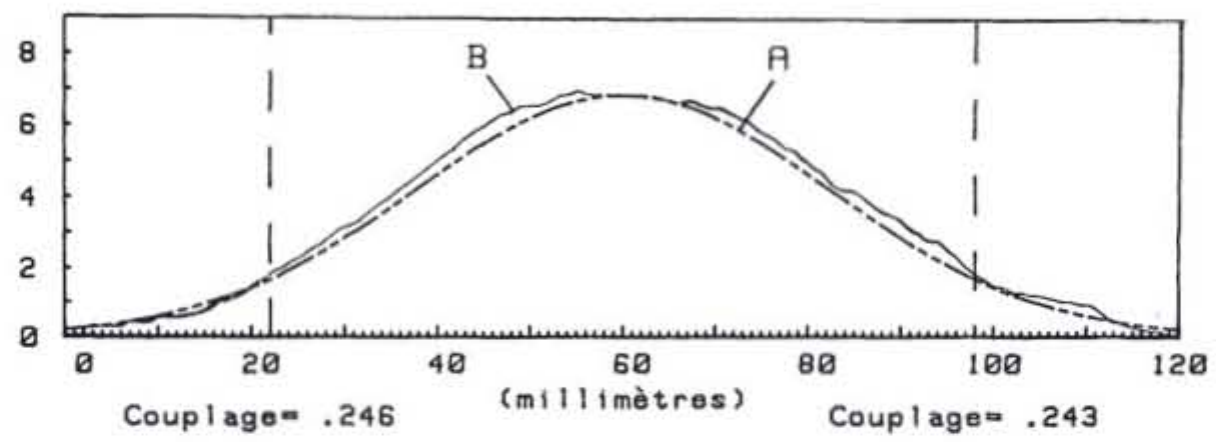

A- Déformation d'un miroir en Molybdène par le moteur central et coefficients de couplage aux points d'appui des deux moteurs voisins. B- Gaussienne.

Fig. 4

D'autres dispositifs ont aussi été réalisés. Parmi ceux-ci, ITEK a réalisé un dispositif constitué d'un bloc de céramique piézo-électrique entre les faces duquel est appliquée une répartition de champ électrique (Fig.5f). Perkin-Elmer $a$, de son côté, expérimenté un miroir formé d'une membrane mince déformée par une répartition de champ électrostatique (Fig.5g).

Plusieurs paramètres contribuent a la conception d'un miroir actif. Parmi ceux-ci, les plus importants sont $1^{\prime}$ amplitude et la rapidité des corrections à effectuer et la longueur transverse de cohérence de l'atmosphère considérée, ces paramètres étant directement liés au domaine de longueur d'onde choisi.

Pour les miroirs constitués d'une plaque mince supportée par des moteurs, la légèreté de la plaque est souvent un paramètre important. Cependant, la plaque ne devra pas se déformer pour $d^{\prime a u t r e s}$ raisons que la solicitation des moteurs qui la supporte (flexion sous son propre poids, pression des outils lors du polissage, ....). Elle devra donc être suffisamment rigide.

Sur la fiqure 5, nous avons représenté schématiquement les divers types de miroirs actifs réalisés. 


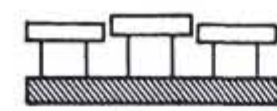

5 a

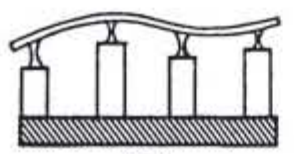

$5 \mathrm{c}$

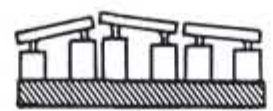

$5 \mathrm{~b}$

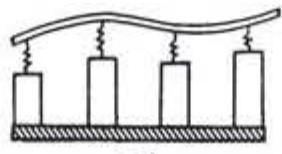

$5 d$
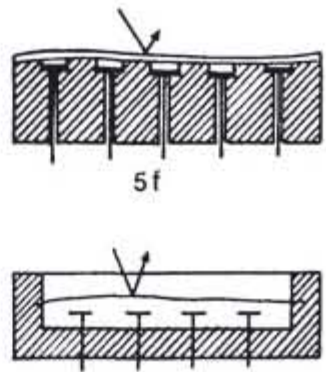

$5 \mathrm{~g}$

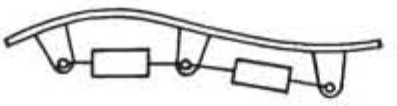

$5 e$

Divers types de miroirs actifs.

Fig. 5

Les moteurs destinés à activer les miroirs peuvent être de plusieurs types suivant 1'application recherchée. Le plus souvent, il s'agit de moteurs mettant en oeuvre les propriétés ferro-électriques de certains matériaux cristallins. Ces matériaux se déforment sous l'action d'un champ électrique. Cette déformation peut être obtenue dans des matériaux piézo-électriques dont les plus utilisés sont les PZT $\left(\mathrm{Pb}(\mathrm{ZrTi}) \mathrm{O}_{3}\right)$ ou des matériaux électrostrictifs tels que les $\mathrm{PMN}\left(\mathrm{Pb}\left(\mathrm{Mg}_{1 / 3} \mathrm{Nb}_{2 / 3}\right) \mathrm{O}_{3}\right)$.

- Les matériaux piézo-électriques. Ils se déforment linéairement avec le champ appliqué. Ils présentent de fortes hystérésis (de l'ordre de 10\%). On les trouve sous différentes formes : disques, empilements de disques, cylindres creux, bilames. Le choix de ces dispositifs dépend de la sensibilité et de la rigidité recherchée. Une solution intéressante consiste en un empilement de disques sur les électrodes desquels les tensions sont adressées sous forme binaire. Dans ces conditions, et puisque les tensions prennent toujours les mêmes valeurs, l'ensemble ne présente plus d'hystérésis.

- Les matériaux électrostrictifs. Ils sont d'apparition plus récente. Leur déformation est proportionnelle au carré du champ appliqué. Ils présente de trés faibles hystérésis ( $2 \%$ mais sont cependant moins sensibles que les matériaux piézo-électriques et trés capacitifs. Ils exigent donc des électroniques de commande capables de fournir de fortes puissances.

En général, la sensibilité à la déformation sous 1'effet du champ appliqué est inversement proportionnelle à la rigidité mécanique du moteur. Ceci nous conduit à caractériser ces moteurs par l'équivalent d'un produit gain-bande passante. Dans certains cas, l'élasticité du moteur peut être mise à profit lorsque le moteur est destiné à guider un miroir continu en $y$ appliquant une 
contrainte plutôt que en lui imposant un déplacement.

Des moteurs tels que des translateurs à vis micrométriques ont aussi été utilisés. Ces moteurs permettent des déplacements de grande amplitude mais souffrent d'un manque de rapidite. Ils sont parfois associés à des moteurs piézo-électriques capables de fournir des déplacements rapides de faible amplitude. En effet, le spectre des fluctuations à corriger est, en général, une fonction décroissante de la fréquence de ces fluctuations.

\section{Dispositifs analyseurs de surface d'onde.}

Compte tenu de la diversité des applications des miroirs adaptatifs, un grand nombre de dispositifs capables de mesurer les défauts à corriger ont été imaginés et utilisés. On peut les classer suivant la nature de la lumière à analyser.

3-a Ondes monochromatiques. Dans ce cas, de nombreuses méthodes interférométriques ont été mises en oeuvre. Généralement, ce type d'analyseur est utilisé lorsqu'il existe une onde de référence, ce qui est le cas des systèmes qui permettent de focaliser un laser sur une cible. L'analyse de la surface d'onde peut être réalisée par interférométrie classique, les franges présentes dans le champ étant analysées point par point et la phase calculée à partir de mesures d'intensité. Elle peut aussi être analysée par des méthodes dynamiques mettant en oeuvre des modulations de phase ou de fréquence ou un décalage en fréquence de $l^{\prime}$ onde de référence. Ces méthodes présentent l'inconvénient de donner des résultats entachés d'ambiguité lorsque la déformation à mesurer est supérieure à la longueur d'onde.

3-b ondes quasi-monochromatiques. Pour l'analyse de telles surfaces d'onde, des interféromètres à contraste de phase du type zernike ou des interféromètres à décalage ont été utilisés. Dans ces systèmes, $1^{\prime}$ onde interfère avec tout ou partie d'elle-même et la cohérence n'est pas exigée bien qu'elle limite les performances du dispositif. Accroftre la cohérence de la lumière à analyser par l'addition d'un filtre implique une réduction de la sensibilité du système.

3-c Lumière blanche. Deux dispositifs de ce type ont été développés. Le premier est basé sur la méthode de Hartmann. Un tel dispositif est représenté sur la figure 6 . 


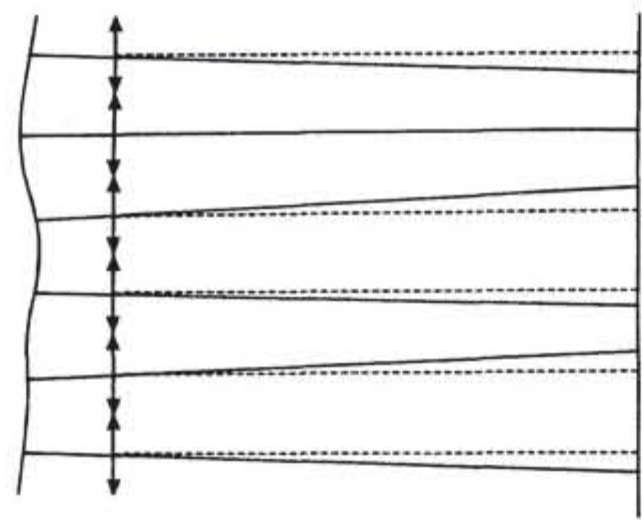

Analyseur de surface d'onde par la méthode de Hartmann.

Fig. 6

La pupille est décomposée en une série de sous-pupilles de dimensions inférieures à $\rho_{0}$ de telle sorte que, dans chaque sous pupille, l'onde ne subisse qu'une déviation. La lumière ayant traversé chaque sous pupille est ensuite focalisée grâce à un réseau de lentilles. La position de la tache ainsi obtenue par rapport à l'axe de la lentille correspondante sera caractéristique de la pente de la portion d'onde considérée. Généralement, la position de ces taches est détectée par une mosalque de détecteurs. Dans certains cas, et dans les systèmes destinés à l'asservissement d'une optique adaptative, des détecteurs à quatre quadrants disposés sur l'axe de chaque sous pupille peuvent être utilisés.

Une autre technique a été aussi mise au point pour l'analyse de lumière incohérente. Il s'agit d'un interféromètre à décalage dynamique. Un tel dispositif est représenté sur la figure 7 . 


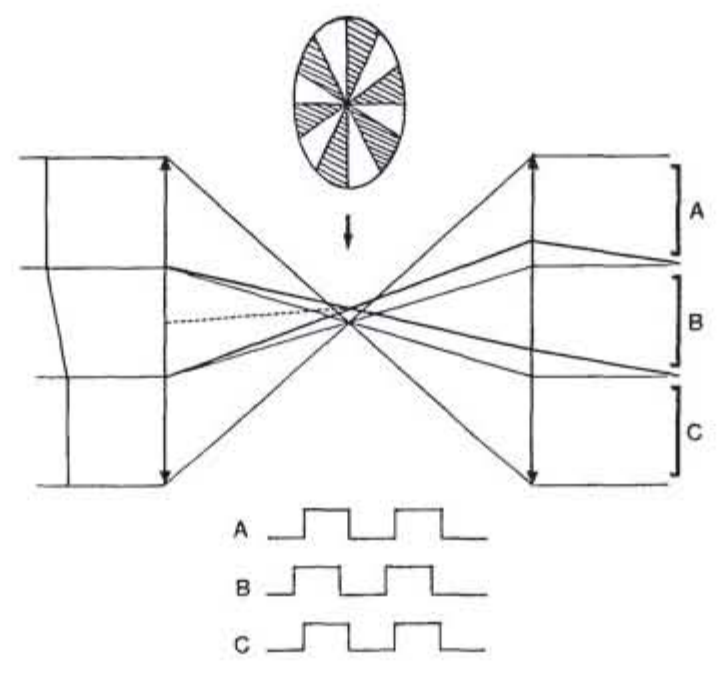

Analyseur de surface d'onde à décalage dynamique.

Fig.7

Comme dans le précédent dispositif, le champ est séparé en un certain nombre de sous pupilles. $L^{\prime}$ image du point à analyser est formée dans un plan intermédiaire P. Dans ce plan, un disque tournant présentant un réseau périodique de zones transparentes et opaques vient hacher l'image. La lumière ayant traversé chaque sous-pupille est ensuite focalisée sur un ensemble de détecteurs. Les portions de surface d'onde arrivant perpendiculairement à l'axe de l'instrument sont focalisées sur cet axe et seront obturées simultanément par le disque tournant. Si la portion $\mathrm{d}^{\prime}$ onde correspondant à une sous pupille n'est pas perpendiculaire à l'axe, sa tache de focalisation sera localisée hors de l'axe et l'avance ou le retard du temps d'obturation de ce point par rapport aux autres sera proportionnelle au gradient de la phase dans cette direction. Naturellement, deux systèmes identiques agissant suivant deux directions perpendiculaires sont nécessaires.

3-d Dispositifs destinés à optimiser l'énergie encerclée. $D^{\prime}$ autres dispositifs, essentiellement destinés à l'asservissement des optiques adaptatives, permettent de maximiser l'énergie encerclée. Ils sont basés sur une méthode action test et réaction. $\mathrm{Ce}$ type de méthode peut être utilisée en séquence. Dans ce cas, chaque portion de miroir est actionné successivement afin de maximiser l'énergie reçue sur un détecteur (Polysteps). Lorsque la correction exige une certaine rapidité, les miroirs peuvent être actionnés simultanément, $1^{\prime}$ influence de chaque zone de miroir sur l'intensité reçue étant distinguée par des fréquences caractéristiques de cette zone. Cette technique peut être utilisée en modulant sinusoïdalement des portions de miroirs à des 
fréquences différentes (Multidithered systems) ou en excitant les modes de vibrations naturels du miroir (Modal Wavefront Control Systems).

Dans le cas où chaque portion de miroir est activée à une fréquence différente, son influence sur l'intensité focalisée est détectée de manière synchrone, la fréquence caractérisant chaque portion étant prise pour référence afin d'extraire du signal total la correction à lui apporter. Dans cette méthode, la plus basse fréquence doit être trés supérieure à la bande passante des asservissements et l'intervalle entre chaque fréquence doit aussi être supérieur à cette bande passante, ceci afin d'éviter les inter-modulations. Cette technique est le plus souvent utilisée dans le cas des miroirs segmentés où l'ensemble miroir-moteur peut être trés rigide et permettre ainsi des fréquences de modulations trés élevées.

Les dispositifs mettant à profit l'excitation des modes propres du miroir peuvent être exploités en analysant la surface d'onde en termes de polynômes de Zernike et en synthétisant numériquement les corrections à apporter à chaque mode correspondant. De tels miroirs doivent être conçus pour que l'espacement entre les fréquences des modes propres de vibration soit compatible avec la bande passante de l'asservissement souhaité.

\section{Conclusion.}

Dans ce bref exposé, nous nous sommes efforcés de décrire les techniques classiques les plus utilisées pour la mise en oeuvre de miroirs actifs et adaptatifs. Compte tenu de la profusion le la littérature portant sur ce sujet, il ne nous a pas été possible de citer tous les auteurs qui ont contribué aux récents progrès dans ce domaine faute de place. Les références des travaux anciens qui sont à l'origine de l'optique active et adaptative peuvent être trouvées dans l'article de revue de John W. HARDY. Pour les travaux plus récents, deux numéros de la revue "Optical Engineering" ont été consacrés, en 1990, à ce sujet.

\section{REFERENCES}

Active Optics : A New Technology for the Control of Light. J. W. HARDY Proceeding of IEEE Vol.66 N॰6 pp 651-694 (June 1978).

optical Engineering Vol $29 \mathrm{~N}^{\circ} 10$ et 11 (Octobre et Novembre 1990). 